\title{
Calidad de Maíz Colorado Flint para Industria Cervecera en Corrientes, Argentina
}

Celsa N. Balbi ${ }^{1}$, Oscar R. Valentinuz ${ }^{2}$ y Juan Prause ${ }^{3}$

(1) Universidad Nacional del Nordeste, Facultad de Ciencias Agrarias, Cátedra de Cultivos I, Sargento Cabral 2131, (3400) Corrientes-Argentina.

(2) Universidad Nacional de Entre Ríos, Facultad de Ciencias Agropecuarias, Cátedra Cereales y Oleaginosas, Ruta 11, km 11,5 , (3101) Oro Verde, Entre Ríos-Argentina

(3) Universidad Nacional del Nordeste, Facultad de Ciencias Agrarias, Cátedra de Agroclimatología, Sargento Cabral 2131, (3400) Corrientes-Argentina

Recibido Mar. 03, 2009; Aceptado Abr. 29, 2009; Versión Final recibida Jun. 26, 2009

\section{Resumen}

El trabajo se realizó con el objetivo de vincular variables de crecimiento y calidad de híbridos de maíz colorado flint usado en a industria cervecera. Se realizó un experimento con 5 híbridos comerciales a una densidad de $6 \mathrm{pl} \mathrm{m}^{-2}$ conducidos sin limitantes hídricas ni nutricionales en un diseño de bloques completos al azar con cuatro repeticiones. Se midió materia seca, índice de área foliar e intercepción de radiación antes y después del período crítico, rendimiento y calidad, índice de flotación, peso hectolítrico, relación de molienda y materia grasa. Se obtuvo rendimientos de 771 y $982 \mathrm{~g} \mathrm{~m}^{-2}(p<0.0001)$ para los diferentes híbridos. Se encontraron asociaciones entre la relación fuente destino posfloración y dos variables de calidad, índice de flotación y peso hectolítrico.

\section{Orange Flint maize Quality to Brewing Industry in Corrientes, Argentina}

\begin{abstract}
This work was carried out with the aim of linking growth and quality variables of orange flint maize hybrids to use in brewing industry. The experiment was settled with five commercial maize hybrids in a randomised complete block design with four replications in random plots of 6 lines and $20 \mathrm{~m}$ long. The experiments were conducted without any limiting factor such as water and nutrients. The variables measured were dry matter content, area index, radiation interception, yield and quality, floating rate, test weight, and relation between milling and fat. Yield obtained for the different hybrids were 771 and $982 \mathrm{~g} \mathrm{~m}-2$ ( $p<0.0001)$. Also, association between the relation source-posflowering destination and two quality variables, the flotation index and the weight test.
\end{abstract}

Keywords: orange flint maize, brewing industry, commercial hybrids, growth and quality 


\section{INTRODUCCION}

Calidad proviene del latín Qualitas que significa atributo, propiedad o naturaleza básica de las cosas (Aguirrezabal y Andrade, 1998). Sin embargo ese atributo o conjunto de atributos deben ser valorados o requeridos en función de su destino. La industrialización de maíz es una de las actividades agroindustriales que genera mayor valor agregado, por su gran número de productos y subproductos generados Según Watson (1988) en Corn marketing, processing and utilization menciona que la industria de la molienda seca exige granos que rindan elevadas proporciones de fracciones gruesas ("grits") que serán destinados a la elaboración de copos para desayuno, a la industria cervecera, sémolas para alimentación humana y harinas diversas (ver Aguirrezabal y Andrade, 1998). El maíz colorado duro, "flint" o "plata argentino" reúne las cualidades, ya que se fragmenta en trozos de mayor granulometría que el maíz dentado y el germen se despega con facilidad del endospermo (Seetharam et al., 2001).

Si bien, el maíz tipo duro predominó durante mucho tiempo en nuestro país, la introducción de cultivares amarillos dentados desde Estados Unidos y Europa fueron desvirtuando los atributos de dureza con el objetivo de lograr mayores rendimientos (Eyherabide et al., 1996). El mejoramiento ha avanzado en el mismo sentido, con una marcada tendencia en la última década hacia la obtención de organismos genéticamente modificados (OGM). En el noreste y noroeste argentino, la Cervecería y Maltería Quilmes, ha incrementado la demanda local de maíz tipo "flint" o "Plata" (Res. SAGPYA 757/97). En la campaña 2007/08 ha demandado $40.000 \mathrm{Mg}$ de este grano aunque desechan por cuestiones de mercado los OGM. Su demanda esta bien definida hacia maíces con índice de flotación (IF) menor al $25 \%$ y bajos porcentajes de grasa, a efectos de que durante su oxidación no modifiquen el sabor de la cerveza (Dimotta, 2006. Comunicación personal).

La propiedad de dureza del grano es de naturaleza intrínseca y se expresa en la resistencia a la acción mecánica y se asocia a la composición bioquímica del endosperma: contenido relativo de las proteínas de reserva -zeínas- y componentes del almidón (amilosa y amilopectina) (Robutti et al., 1997; Robutti at al., 2000). La dureza es una característica fundamentalmente heredable, modificada por las condiciones de cultivo, su ambiente y humedad de cosecha (Borrás et al., 2002; Cirilo et al., 2003). Un test para poder detectarla fácilmente es el test del índice de flotación. El noreste argentino presenta condiciones ambientales con un régimen de altas temperaturas y precipitaciones con lo cual es de esperar modificaciones en ese sentido. Las altas temperaturas durante las fases tempranas de desarrollo del endosperma reprimen la acumulación del zeínas al nivel de su síntesis. A su vez, la acumulación de zeínas parece ser reprimida principalmente por degradación de la proteína, aparentemente por una progresión natural de desarrollo del grano y no por algún efecto significativamente claro de estrés por calor durante este proceso, aunque no se han encontrado diferencias significativas en los tratamientos con temperaturas altas (Monjardino et al., 2005).

En estas condiciones ambientales, todas las etapas tienden a acortarse por efecto de las temperaturas y aunque el fotoperíodo alcance valores mayores que los de latitudes mas altas (Andrade et al., 1996), las altas temperaturas no permiten manifestar diferencias en longitud de ciclo en días cronológicos. Aunque seria de esperar modificaciones en la expresión de los diferentes genotipos respecto de la generación del rendimiento y la calidad. Este cultivo, de calidad diferenciada se torna aún más promisorio con la creciente alternativa de realizar doble cultivo primavero-estival en el noreste argentino, para lo cual hay que evaluar su potencial en siembras tardías.

El tamaño del grano es una característica comercialmente relevante. Factores de estrés que reducen el crecimiento de las plantas durante el llenado de los granos, limitan la provisión de asimilados a los mismos. La cantidad de biomasa acumulada en la planta por cada grano durante su llenado (relación fuente-destino) determina el peso final que puede alcanzar (Cirilo y Andrade, 1996; Borrás y Otegui, 2001) cuestión que se enfatiza en maíces dentados (Gambín et al., 2006). Cuando existen limitaciones al crecimiento del cultivo durante el llenado de los granos se reduce también la energía disponible para la absorción y reducción de nitrógeno y azufre desde el suelo (Uhart y Andrade, 1995). En consecuencia, el flujo de nitrógeno y azufre hacia los granos se reduciría en mayor medida que el de carbohidratos, disminuyendo la concentración de nitrógeno y azufre en el grano a la 
cosecha, lo que afectaría su dureza. Por otro lado, el peso hectolítrico es un factor mas estable ya que se mantiene aún con defoliaciones en la planta (Thomison y Geyer, 2006).

El mercado de los semilleros ofrece pocos híbridos que ofrezcan características de dureza y que estén adaptados a la región del noreste argentino y, algunos, a pesar de haber sido liberados al mercado hace muchos años, aún no fueron estudiados respecto de su respuesta a condiciones ambientales y manejo tecnológico. Algunos de ellos, durante su mejoramiento se han transformado genéticamente desde duros colorados con endosperma córneo de corona lisa y color rojizoanaranjado a presentar zonas amarillentas en la corona y el endosperma del grano lo que repercute en una menor dureza. Otros, poseen una aparente inestabilidad en sus atributos de calidad, lo que al momento de comercializar se refleja en el IF, lo cual no es apreciado por la industria cervecera (Dimotta, 2006. Comunicación personal).

El presente trabajo tiene como objetivo determinar el efecto del ambiente productivo del noreste argentino en el crecimiento y la generación de la calidad de cinco híbridos de maíz colorado duro o flint con diferente trasfondo genético, durante el período critico, 30 días centrados en floración y vincularlo con la generación de la calidad.

\section{MATERIALES Y METODOS}

El ensayo se realizó en la localidad de Corrientes, en el Campo Experimental de la Facultad de Ciencias Agrarias de la UNNE. Los datos del presente trabajo son los del experimento realizado durante el ciclo agrícola 2006 - 2007. El Campo Experimental está ubicado en las siguientes coordenadas geográficas: latitud: $27^{\circ} 28^{\prime} 27.23^{\prime \prime}$ S; longitud: $58^{\circ} 47^{\prime} 00.66^{\prime \prime} \mathrm{O}$; y altitud: $50 \mathrm{~m}$ sobre el nivel del mar. El suelo esta clasificado como Udipsament acuico hipertérmico de la serie Ensenada Grande (Escobar et al., 1996).

Se utilizaron cinco híbridos comerciales provenientes de distintas empresas seleccionados en función de tres aspectos: i) Híbridos más demandados actualmente por la industria cervecera local: Avant Syngenta y Pioneer 3041, ii) Híbridos con característica de típico colorado flint: FR Don Luna Fortín Refugio y iii) Híbridos con mayor potencial de rendimiento (nuevos en el mercado): Pioneer $30 \mathrm{P} 70$ y DK 910 Monsanto.

El 8 de enero de 2007 se sembraron cinco híbridos de maíz tipo duro colorado en parcelas de 6 líneas a $52 \mathrm{~cm}$ de $20 \mathrm{~m}$ de largo con cuatro repeticiones para cada hibrido, a una densidad de siembra de una planta cada $30 \mathrm{~cm}$ (64000 plantas ha $\left.{ }^{-1}\right)$. El experimento se condujo sin limitaciones hídricas ni nutricionales, el riego se realizó por un sistema de goteo y fertilización a la siembra con $100 \mathrm{~kg} \mathrm{ha}^{-1}$ de fertilizantes combinados (NPKS 11-17-21-8) y $100 \mathrm{~kg} \mathrm{ha}^{-1}$ de fertilizante nitrogenado (NPK 33-0-0) en las fases vegetativas V4, V6 y V8.

Se registraron los datos climáticos de temperatura y radiación solar en las condiciones del experimento con un Data Logger de 8 canales (Empresa Cavadevices, Buenos Aires, Argentina), con sensores de radiación PAR, compuesto por una celda de GaAsP con respuesta espectral en la banda comprendida entre los 400 y los $700 \mathrm{~nm}$ de longitud de onda y dos de temperatura (aire y suelo de -40 a $+125^{\circ} \mathrm{C}$ ). Las precipitaciones fueron registradas con la estación meteorológica Weather Monitor II de Davis SA.

Se realizaron determinaciones de estadíos fenológicos (Escala de Ritchie y Hanway, 1982) para ello se marcaron al azar 10 plantas en cada parcela de cada repetición. Para el cálculo de tiempo térmico de aparición de hojas se utilizó la formula: TT $=\Sigma$ ( $\mathrm{T}$ amb $-\mathrm{T}$ base $)$ donde: $\mathrm{T}$ amb es la media diaria y T base se utilizó $10^{\circ} \mathrm{C}$.

Para las mediciones fenológicas se tomaron en cuenta las siguientes fechas, i) $50 \%$ de emergencia, ii) floración (el $50 \%$ de plantas con estigmas visibles) sobre 10 plantas de la parcela y iii) madurez fisiológica. Se determinó la formación de capa negra en la base de los granos. Los muestreos se realizaron cada tres días considerando 5 granos por espiga de 6 espigas por unidad experimental, marcadas a tal efecto. 
Se realizaron muestreos de planta entera en tres momentos del ciclo del cultivo, R1 menos 15 días, R1 más 15 días y madurez fisiológica, en cada uno se cortaron a ras del suelo dos metros lineales de los dos surcos centrales. Las plantas se seccionaron en tallo más vaina más panoja, hojas, chalas y espiga cuando existían. Luego se secaron en estufa a $70^{\circ} \mathrm{C}$ hasta peso constante. Cada sección de la planta se pesó con balanza Denver XP300 (300-0.1 gr) o Ohaus CS2000 (2000-1 gr) por separado para registro de la partición de asimilados.

Para la medición de la intercepción de radiación solar por el cultivo (Ei) se tomaron los valores de radiación fotosintéticamente activa (RFA) interceptada por el cultivo utilizando un sensor lineal quantum (Cavadevices, B.A, Argentina). Se realizaron 5 mediciones por parcela ubicadas desde el centro de un entresurco hasta el centro del entresurco contiguo, entre las 11.30 y 13.00 hs, en días soleados, de acuerdo a la metodología propuesta por Gallo y Daughtry (1986). El porcentaje de RFA interceptada por el cultivo se calculó como [1 - (It /I0)] x 100 donde It es la RFA medida entre el suelo y la primera hoja verde, e 10 es la RFA incidente por encima del cultivo. La eficiencia de conversión de la radiación $(\mathrm{Ec})$ se calculó como el cociente entre materia seca producida por el cultivo y la RFA interceptada acumulada por el cultivo para una determinada etapa.

Se realizaron mediciones de radiación interceptada en $\mathrm{R} 1$ menos 15 días, luego de este momento cada 7 días aproximadamente, se hizo coincidir una medición con R1 más 15 días y con madurez fisiológica.Se realizaron mediciones de área foliar en 5 plantas de cada parcela en los siguientes momentos: i) R1 menos 15 días, ii) R1 más 15 días y iii) madurez fisiológica con el medidor de área foliar portátil LI-COR 3000. En cada una de las parcelas se dejaron los dos líneos laterales y dos metros lineales entre muestreos para eliminar el efecto bordura.

Cuando los granos tuvieron una humedad de aproximadamente $25 \%$ se cosecharon las espigas de cuatro metros lineales de los dos líneos centrales de cada parcela. El peso seco de grano se calculó contando 1000 granos que fueron secados en estufa con circulación forzada de aire $\left(65^{\circ} \mathrm{C}\right)$ hasta peso constante. El número de granos $\mathrm{m}^{-2}$ se calculó mediante el cociente entre rendimiento de grano (sobre base seca) y el peso del grano. Se acondicionaron $5 \mathrm{~kg}$ del grano cosechado por híbrido para la determinación los análisis de calidad realizados en el Laboratorio de Calidad de Cereales de la Estación Experimental Agropecuaria Pergamino del Instituto Nacional de Tecnología Agropecuaria. El peso hectolítrico fue determinado en muestras de aproximadamente $400 \mathrm{~g}$ usando un instrumento Tripette \& Renaud TR-400. Valores de peso hectolítrico más altos están generalmente asociados con maíces más duros según lo expresado por Watson (1988) en Corn marketing, processing and utilization (ver Aguirrezabal y Andrade, 1998). La materia grasa fue determinada con el método Soxhlet (A.O.A.C., 1990)

El índice de flotación fue determinado según Lepes et al. (1976). Este método fue una modificación del método de Wichser (1961), en el que 100 granos enteros y sanos fueron colocados dentro de un vaso de precipitado de $250 \mathrm{ml}$, conteniendo aproximadamente $170 \mathrm{ml}$ de una mezcla de tetracloruro de carbono y kerosén. Las proporciones de estos compuestos se ajustaron hasta alcanzar densidad de la mezcla de $1,305 \mathrm{~g} / \mathrm{cm} 3$ a $25^{\circ} \mathrm{C}$. Después de ser brevemente agitados con una varilla de vidrio, los granos que flotan son contados. Menor número de granos que flotan indican una mayor dureza.

La relación de molienda fue determinada de manera similar a aquella descripta por Pomeranz et al. (1986) para la relación de peso gruesos/finos (G/F). Muestras de $50 \mathrm{~g}$ fueron molidas durante $15 \mathrm{seg}$. en un molino de laboratorio Stein. Los granos molidos fueron tamizados a velocidad máxima por 1 min. en un tamizador mecánico Chopin Rotachoc equipado con tamices circulares de $1 \mathrm{~mm}$ y 0,5 $\mathrm{mm}$. de apertura de malla. Cantidades de material grueso retenido por el tamiz de $1 \mathrm{~mm}$ y de material fino que pasa a través del tamiz de $0,5 \mathrm{~mm}$ fueron pesados con una precisión de $0,1 \mathrm{~g}$. La relación entre los pesos de las respectivas fracciones es la relación G/F. La relación es más alta para maíces más duros.

Los tratamientos del experimento se distribuyeron con un diseño de bloques completos al azar con cuatro repeticiones. Los datos se analizaron mediante análisis de variancia y las asociaciones entre variables mediante análisis de correlación y regresión. Los análisis se realizaron con el Programa Infostat, Versión 1.1. 


\section{RESULTADOS Y DISCUSION}

La Tabla 1 muestra los rendimientos en grano obtenidos con valores entre 771 y $982 \mathrm{~g} \mathrm{~m}^{-2}$ $(p<0.0001)$ para los híbridos ensayados. En este trabajo, el cultivo conducido sin limitantes hídricas ni nutricionales, permitió la obtención de los rendimientos máximos; expresando todo su potencial. El rendimiento potencial de un híbrido o cultivar ha sido definido como el rendimiento obtenido cuando crece bajo condiciones no limitantes (hídricas y nutricionales) en un ambiente al cual se encuentra adaptado (Andrade al., 1996).

Tabla 1: Componentes numéricos del rendimiento: Rendimiento en grano $\left(\mathrm{g} \mathrm{m}^{-2}\right)$, peso 1000 granos (g), número de granos calculado. Letras distintas indican diferencias significativas $(p<=0,05)$

\begin{tabular}{|l|c|c|c|}
\hline & \multicolumn{3}{|c|}{ COMPONENTES NUMERICOS DEL RENDIMIENTO } \\
\hline HIBRIDOS & RENDIMIENTO & PESO 1000 GR & N $^{\circ}$ DE GRANOS \\
\hline AVANT & $801 \mathrm{a}$ & $366,0 \mathrm{c}$ & 1919,8 \\
\hline 30P70 & $982 \mathrm{~b}$ & $320,1 \mathrm{~b}$ & 2691,0 \\
\hline DK 910 & $824 \mathrm{a}$ & $331,0 \mathrm{~b}$ & 2183,7 \\
\hline DON LUNA & $771 \mathrm{a}$ & $293,4 \mathrm{a}$ & 2305,1 \\
\hline P3041 & $818 \mathrm{a}$ & $362,0 \mathrm{c}$ & 1982,2 \\
\hline
\end{tabular}

Los efectos de la temperatura sobre el desarrollo y de la radiación solar sobre la acumulación de biomasa definen los límites de los rendimientos (Andrade et al., 1996). De esto surge que los rendimientos obtenidos indicarían la potencialidad en términos de rendimiento en siembras de enero o de segunda, demostrando también que altos niveles de rendimiento pueden lograrse con maíces colorados. El híbrido mas destacado fue Pioneer $30 \mathrm{P} 70$ con $982 \mathrm{~g} \mathrm{~m}^{-2}$, aunque con menor calidad para su uso en industria cervecera.

En la Tabla 2 se observa que no se encontraron diferencias significativas entre los híbridos en términos de eficiencia de conversión (Ec) o eficiencia de uso de la radiación (EUR), sin embargo el híbrido con la mayor Ec (P3041) mostró ventajas en el índice de flotación y peso hectolítrico $(p<0.05)$, destacándose como el de mejor calidad junto con DK 910 que tiene menor contenidos en grasas.

Tabla 2: Componentes ecofisiológicos del rendimiento: Materia seca R1 - 15 días (g), Materia seca $\mathrm{R} 1$ + 15 días $(\mathrm{g})$, eficiencia de conversión de la radiación $\left(\mathrm{g} \mathrm{Mj}^{-2}\right.$ dia). Letras distintas indican diferencias significativas $(p<=0,05)$

\begin{tabular}{|l|c|c|c|c|}
\hline & \multicolumn{4}{|c|}{ COMPONENTES ECOFISIOLOGICOS DEL RENDIMIENTO } \\
\hline HIBRIDOS & $\begin{array}{c}\text { MATERIA } \\
\text { SECA R1 }-15\end{array}$ & $\begin{array}{c}\text { MATERIA SECA } \\
\text { R1 }+15\end{array}$ & $\begin{array}{c}\text { EFICIENCIA } \\
\text { CONVERSION }\end{array}$ & $\begin{array}{c}\text { RELACION FD } \\
\text { POSFLORACION }\end{array}$ \\
\hline AVANT & 247,47 & 1057,52 & $2,46 \mathrm{a}$ & $0,49 \mathrm{c}$ \\
\hline 30P70 & 264,5 & 1107,23 & $2,59 \mathrm{a}$ & $0,36 \mathrm{a}$ \\
\hline DK 910 & 239,14 & 1038,70 & $2,64 \mathrm{a}$ & $0,42 \mathrm{~b}$ \\
\hline DON LUNA & 191,20 & 1127,98 & $2,72 \mathrm{a}$ & $0,41 \mathrm{~b}$ \\
\hline P3041 & 272,31 & 1201,85 & $2,81 \mathrm{a}$ & $0,53 \mathrm{c}$ \\
\hline
\end{tabular}

Los valores de Ec encontrados desde 2,46 a 2,81 $\mathrm{g} \mathrm{Mj} \mathrm{m}^{-2} \mathrm{dia}^{-1}$ para los híbridos ensayados son similares a los obtenidos en Balcarce con valores promedios de 2,77 $\mathrm{g} \mathrm{Mj} \mathrm{m}^{-2} \mathrm{dia}^{-1}$ (Andrade, 1995), algo menores a los esperados. En efecto, las expectativas de alcanzar valores mas elevados de Ec en esta región, se fundamentaban en que la eficiencia fotosintética del maíz (planta C4) aumenta con la temperatura con un óptimo entre 30 y $40^{\circ} \mathrm{C}$ (Fischer y Palmer, 1984; Hay y Walker, 1989). Los maíces colorados flint, cuyo contenido en proteínas es mayor al de los maíces dentados cultivados en Balcarce, podría explicar al menos parcialmente, un mayor gasto energético de la planta durante el período reproductivo con la consiguiente caída de Ec.

En la Tabla 3 se reflejan los índices de flotación (IF) y contenido de grasa, ambas características muy valoradas en la industria cervecera. Los IF reflejan valores entre 0,75 y $9,5(p<0.008)$, que califican 
para su uso en la industria cervecera (Res. SAGPYA 757/97), a pesar de que algunos híbridos muestran mejor comportamiento que otros. El contenido de grasa está expresado en porcentaje sobre materia seca de grano en grano entero, los resultados muestran una fuerte vinculación entre genotipo y contenido de grasa con resultados que van desde 4.55 a $5.94 \%$ con diferencias significativas $(p<0,0001)$ y $R^{2}=0.93$, los contenidos de grasa están vinculados principalmente al tamaño del embrión, parte que es eliminada durante el procesamiento del maíz para ser utilizado como adjunto en la industria cervecera. Según lo expuesto por Watson (1988) en Corn marketing, processing and utilization (ver Aguirrezabal y Andrade, 1998) tanto el peso hectolítrico como la relación de molienda son indicadores de dureza de grano, en este experimento el hibrido P3041 mostró el mayor peso hectolítrico, aunque no fue el de mejor comportamiento en la relación de molienda, sin embargo los valores de índice de flotación lo colocan en el mejor lugar.

Tabla 3: Índices de calidad para industria cervecera: Peso hectolítrico (g), Índice de flotación (\%), contenido de grasa $(\%)$ y relación de molienda $(\%)$. Letras distintas indican diferencias significativas $(p<=0,05)$

\begin{tabular}{|l|c|c|c|c|}
\hline & \multicolumn{4}{|c|}{ INDICES DE CALIDAD } \\
\hline HIBRIDOS & $\begin{array}{c}\text { PESO } \\
\text { HECTOLITRICO }\end{array}$ & $\begin{array}{c}\text { INDICE DE } \\
\text { FLOTACION }\end{array}$ & GRASA & $\begin{array}{c}\text { RELACION DE } \\
\text { MOLIENDA }\end{array}$ \\
\hline AVANT & $80,31 \mathrm{a}$ & $5,5 \mathrm{bc}$ & $4,79 \mathrm{a}$ & $4,65 \mathrm{~b}$ \\
\hline 30P70 & $80,55 \mathrm{a}$ & $9,5 \mathrm{c}$ & $5,43 \mathrm{~b}$ & $3,93 \mathrm{a}$ \\
\hline DK 910 & $81,30 \mathrm{ab}$ & $3,0 \mathrm{ab}$ & $4,55 \mathrm{c}$ & $4,03 \mathrm{a}$ \\
\hline DON LUNA & $82,10 \mathrm{bc}$ & $3,0 \mathrm{ab}$ & $5,94 \mathrm{a}$ & $3,60 \mathrm{a}$ \\
\hline P3041 & $82,43 \mathrm{c}$ & $0,75 \mathrm{a}$ & $5,29 \mathrm{~b}$ & $3,83 \mathrm{a}$ \\
\hline
\end{tabular}

Las figuras 1 y 2 muestran las asociaciones entre los indicadores de dureza, índice de flotación y peso hectolítrico, y la relación fuente destino posfloración (gramos de fuente en $\mathrm{R} 1+15$ por grano final).

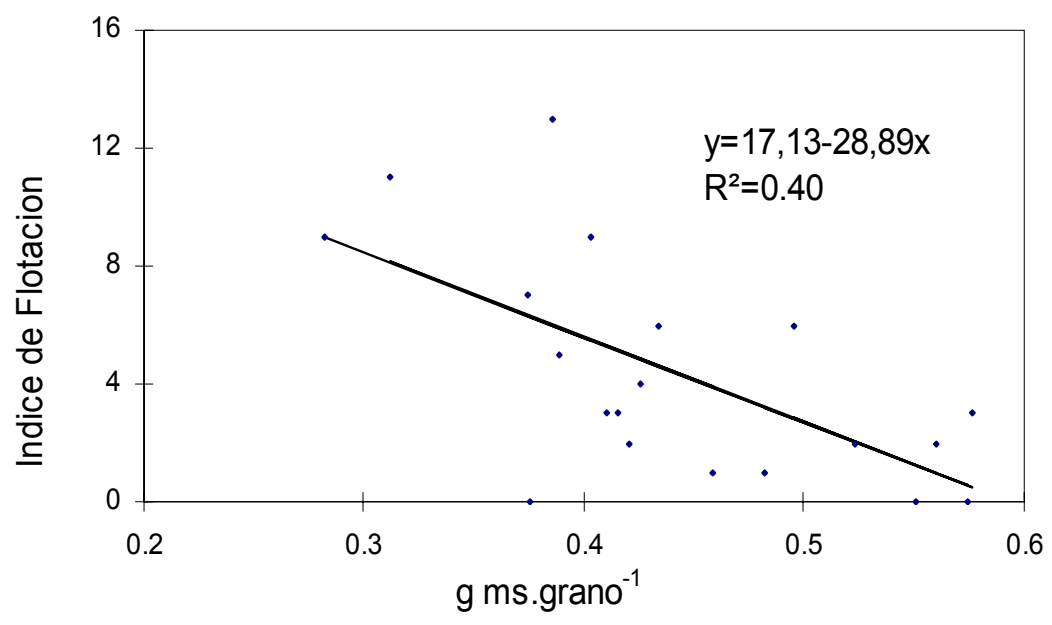

Fig 1: Relación entre fuente destino en R1 + 15 días y el Índice de Flotación

En la Fig 1 se observa que el 40\% del índice de flotación, indicador de dureza de grano, se explica por los g MS/ grano, o sea la relación fuente destino 15 días después de floración. Las estimaciones de los parámetros de la ecuación de regresión resultaron significativos $(p<0.0001)$. En la Figura 2 se observa claramente una alta dispersión. El estimador de beta resultó no significativo $(p=0.2423)$ y el $\mathrm{R}^{2}$ es bajo, indicando que no existe una relación lineal entre ambas variables. Pero si se separan según el tipo de hibrido entre modernos, antiguos (mas comprados en industria) y típico flint este ultimo tipo muestra un $\mathrm{R}^{2}=0.32(p=0.001)$, de este análisis surge que los diferentes genotipos usan diferentes estrategias para lograr una calidad aceptada por la industria. 


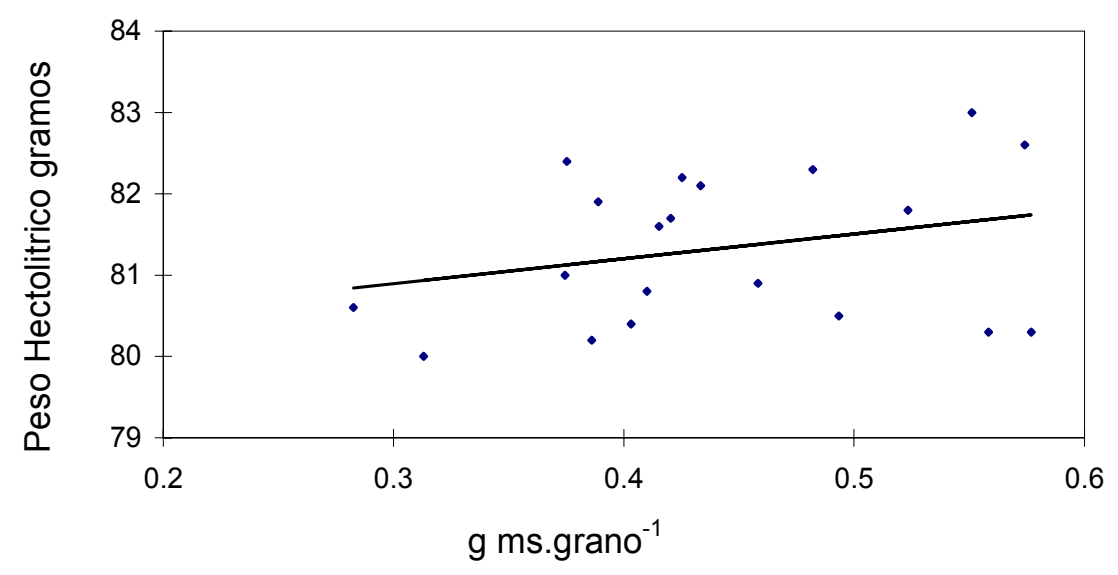

Fig 2: Relación entre fuente-destino en R1 + 15 días y el peso hectolítrico.

Esta vinculación entre crecimiento y la generación de la calidad encontrada fue similar a la hallada en Balcarce (Cirilo et al., 2003), aunque no en una relación lineal simple sino lineal en meseta. Esto sugiere que, en el ambiente productivo del noreste argentino en siembras tardías, el maíz responde de similar manera que en Balcarce respecto de la relación entre el crecimiento en el período reproductivo y la generación de la calidad de granos. EL peso hectolítrico es una característica mas estable (Thomison y Geyer, 2006) y es por ello que no se evidencian respuestas a modificaciones en la relación fuente destino.

\section{CONCLUSIONES}

Se concluye que: i) el maíz colorado flint sembrado en enero tiene un rendimiento potencial que oscila entre los 771 a $982 \mathrm{~g} \mathrm{~m}-2$, y su eficiencia de conversión muestra similares valores que para los maíces no flint, de zonas templadas; ii) su crecimiento en el período reproductivo modifica el índice de flotación y el peso hectolítrico, aunque este último solo como estrategia de un tipo de hibrido. Estos son los principales indicadores de calidad requeridos por la industria cervecera; iii) en fecha tardía, es posible hallar híbridos que muestren buen desempeño tanto en rendimiento como en calidad, debiéndose explorar nuevas combinaciones entre híbridos y las prácticas de manejo del cultivo.

\section{REFERENCIAS}

Aguirrezabal, L. y F. Andrade; Calidad de productos agrícolas. Bases ecofisiológicas, genéticas y de manejo agronómico. INTA, FCA UNMP. 336 p. (1998).

Andrade, F.H. y otros tres autores; Ecofisiología del Cultivo de Maíz. Editorial La Barrosa. 292 p. Buenos Aires. Argentina (1996).

A.O.A.C.-ASSOCIATION OF OFFICIAL ANALYTICAL CHEMISTS Official methods of analysis of AOAC International. 16.ed. Washington, 1995. 1141p.

Borras L. y M.E. Otegui. Maize kernel weight response to post-flowering source-sink ratio. Crop Sci.: 49, 1816-1822 (2001).

Borrás L., J.A. Curá y M.E. Otegui; Maize kernel composition and post-flowering source-sink ratio. Crop Science: 42, 781-790 (2002).

Cirilo A.G. y F.H. Andrade; Sowing date and kernel weight in maize. Crop Science: 36, 325-331 (1996). 
Cirilo, A.G., A. Masague y W. Tanaka; Influencia del manejo del cultivo en la calidad del grano de maíz colorado duro. Revista de Tecnología Agropecuaria. INTA Pergamino: VIII(24), 6-9 (2003).

Dimotta, L.; Maestro Cervecero Empresa Maltería y Cervecería Quilmes SA. Comunicación Personal (2006).

Escobar, E. y otros cuatro autores; Mapa de Suelo de la Provincia de Corrientes. INTA Corrientes (1996).

Eyherabide, G.H., J.L. Robutti, y F.S. Borrás; Effect of Near-Infrared Transmission-Based Selection on Maize Hardness and the Composition of Zeins. Cereal Chem.: 73, 775-778 (1996).

Fischer, K.S. y A.F.E. Palmer; Tropical maize. In Goldsworthy P.R. y N.M. Fisher, eds. The physiology of tropical field crops, p. 213-248. New York, NY, USA, J. Wiley \& Sons (1984).

Gallo, W.P. y C.S.T. Daughtry; Techniques for measuring intercepted and absorbed photosynthetically active radiation in crop canopies. Agron. J.: 78, 752-756 (1986).

Gambín, B.L., L. Borrás y M.E Otegui; Source-sink relations and kernel weight differences in maize temperate hybrids. Field Crops Res.: 95, 316-326 (2006).

Hay, R.K.M. y A.J. Walker; An introduction to the physiology of crop yield. Essex, UK, Longman Scientific and Technical (1989).

Lepes, I.T., R.M. Miotto, A.V. Cedro y O.E. Ruegg; Test de flotación en maíces duros argentinos. I Congreso Nacional de Maiz. 287-298. Pergamino, Argentina (1976).

Monjardino, P., A.G. Smithb y R.J. Jonesa; Heat Stress Effects on Protein Accumulation of Maize Endosperm. Crop Science Society of America: 45, 1203-1210 (2005).

Pomeranz, Y., G.E. Hall, Z. Czuchajowska y F.S. Lai; Test weight, hardness, and breakage susceptibility of yellow dent corn hybrids. Cereal Chem.: 63, 349-351 (1986).

Ritchie S.W. y J.J. Hanway; How a corn plant develops. Special Report 48. Cooperative Extension Service, lowa State. University of Science and Technology. (1982).

Robutti, J.L., F.S. Borrás y G. H. Eyhérabide; Zein composition of mechanically separated coarse and fine portions of maize kernels. Cereal Chem.: 74, 75-78 (1997).

Robutti, J.L., F.S. Borrás, M.E. Ferrer y J.A. Bietz; Grouping and identification of Argentine maize races by chemometric analysis of zein RP-HPLC data. Cereal Chem.: 77, 91-95. (2000)

SAGPyA, Secretaría de Agricultura, Ganadería, Pesca y Alimentación de la Nación. Resolución No757/97, http://infoleg.mecon.gov.ar, Acceso: 15/01/2007.

Seetharam, K. y otros cinco autores; Thermal and functional characterization of starch from Argentinean corn. Cereal Chem.: 78, 379-386 (2001).

Thomison, P. y A. Geyer; Impact of Defoliation on Grain and Stalk Quality in Corn. ASA-CSSA-SSSA International Annual Meetings (2006).

Uhart, S.A. y F.H. Andrade; Nitrogen and Carbon Accumulation and Remobilization during Grain Filling in Maize under Different Source/Sink Ratios. Crop Sci.: 35, 183-190 (1995).

Wichser, W.R.; The world of corn processing. Am. Miller Process: 89(4), 29-31 (1961). 